\title{
Numerical relativity simulations in the era of the Einstein Telescope
}

\author{
Mark Hannam • Ian Hawke
}

Received: date / Accepted: date

\begin{abstract}
Numerical-relativity (NR) simulations of compact binaries are expected to be an invaluable tool in gravitational-wave (GW) astronomy. The sensitivity of future detectors such as the Einstein Telescope (ET) will place much higher demands on NR simulations than first- and second-generation ground-based detectors. We discuss the issues facing compact-object simulations over the next decade, with an emphasis on estimating where the accuracy and parameter space coverage will be sufficient for ET and where significant work is needed.
\end{abstract}

Keywords Numerical relativity · Black Holes · Neutron Stars · Einstein Telescope

PACS 04.25.Dm - 04.30.Db

\section{Introduction}

Experiments aimed at the first direct detection of gravitational waves are now underway with a network of laser-interferometric gravitational-wave (GW) detectors [1-3]. At the completion of the current science run the LIGO and Virgo detectors will be upgraded to second generation detectors, with an order of magnitude improvement in sensitivity, and Advanced LIGO is due to go online in 2014. It is hoped that the second-generation detectors will yield enough observations to begin in earnest the field of GW astronomy; see [4] for an overview of the astrophysics and cosmology potential of GW observations.

Mark Hannam

Physics Department, University College Cork, Cork, Ireland

Faculty of Physics, University of Vienna, Boltzmanngasse 5, A-1090 Vienna, Austria

Ian Hawke

School of Mathematics, University of Southampton, SO17 1BJ, UK 
With the goal of achieving a further factor of ten improvement in sensitivity, combined with an extension of the detector bandwidth to the range $1 \mathrm{~Hz}$ to $10 \mathrm{kHz}$, a design study for a third generation detector has been supported within the European FP7 framework. If these design goals are met, the Einstein Telescope (ET) would be sensitive to a volume of the universe one million times larger than current ground-based detectors, and allow a precision probe of gravitational-wave sources throughout the universe to cosmological distances.

Extracting the most science from ET observations will require accurate theoretical knowledge of GW sources - far more accurate than that required for first- and second-generation detectors. One important aspect of source modelling is the numerical solution of Einstein's equations for the inspiral and merger of compact bodies (black holes and neutron stars) and the numerical simulation of the physics of single compact bodies (neutron star oscillations, instabilities and collapse, and core-collapse supernovae amongst others).

NR simulations have advanced significantly in the last few years, particularly in describing the last orbits and merger of systems of two black holes (see [5-7] for the original breakthrough results, and [8] for an overview of the status of current simulations focused on GW detection). The modeling of neutronstar binaries has also made significant progress from the first simulations of the Tokyo group ([9]) to the current status as recently reviewed by [10].

In this paper we will discuss some of the issues that numerical-relativity simulations face in producing the results needed for ET data analysis and astrophysics applications.

For black-hole binary simulations, the situation is easy to state: since the total mass of the system provides an overall scale to the numerical results, the increased bandwidth of ET does not change the physical nature of the simulations that need to be produced; the black-hole binary parameter space remains the same. However, it does change the accuracy requirement of the simulations, and of the model of the GW parameter-space that can be produced from them.

For matter simulations, the situation is quite different. The scales are set by the physics included in the model. The size of the parameter space is dominated by the unknown physics which it is hoped that GW observations will constrain. In order to make simulations practical, only the "essential" physics will be included in the model. The determination of "essential" is balanced between current knowledge and expectations of the key physics, and what can practically be simulated and detected. Thus the model, resulting parameter space and accuracy requirements will change with the increased bandwidth of ET.

In Section 2 the status of vacuum simulations of binary black holes is discussed, including a discussion of the waveform accuracy of current and nearfuture simulations, the sampling of the parameter space, the construction of template banks and possible future improvements in numerical techniques. Section 3 discusses the situation with matter simulations, assuming that the physical model is "added on" to the spacetime simulation and GWs extracted 
using the same techniques. Section 4 summarises the likely status of NR over the next decade.

\section{Black-hole binaries}

Fully general-relativistic simulations of the dynamics of black-hole binary systems during their last orbits and merger have been possible since 2005 [5-7]. In these simulations initial data are prescribed for a slice of spacetime that contains two black holes, usually in orbit, and in many cases with initial parameters chosen, or tuned, such that the black holes are following non-eccentric quasi-circular orbits [11-13]. The initial data must also satisfy four constraint equations in order to be part of a valid solution of Einstein's equations. The data are advanced to future time slices via a set of evolution equations; the specific equations depend on the choice of decomposition of the four-dimensional Einstein equations to $3+1$ (space+time) dimensions, and the coordinates and time-slicing at successive evolution steps depend on the choice of gauge conditions. We are in principle free to choose the gauge conditions as we wish (as a result of the coordinate invariance of Einstein's equations), but in practice are limited to choices that lead to sufficiently stable and accurate numerical simulations. The issues involved in finding a numerically well-posed and stable formulation of Einstein's equations, a convenient geometrical representation of a black-hole spacetime, construction of black-hole-binary initial data, and numerically and geometrically well-behaved gauge conditions, are discussed in the textbook [14], and in the review articles [15-17].

The parameter space of inspiraling black-hole binaries consists of eleven parameters: the total mass and mass ratio of the system (or alternatively the individual masses of the two black holes), the spin vector of each black hole, the eccentricity of the system, the initial separation of the binary, and the initial phase of the binary orbit. Since the total mass provides an overall scale for the solution, this can be removed from the parameter space of necessary numerical waveforms. If the numerical late-inspiral-plus-merger waveform is combined with an analytic inspiral waveform (for example, calculated from the post-Newtonian approximation), the initial separation of the binary can be made arbitrarily large, and yet one more parameter can be removed from the parameter space. Assuming that the process of constructing a "hybrid" waveform is sufficiently robust (and this remains a topic of current research), we are left with a total of nine parameters. In non-eccentric inspirals, the initial phase has no physical meaning, and can also be removed from the parameter space, so that non-eccentric binaries consist of a seven-dimensional parameter space, i.e., only the mass ratio and spin components.

To date late-inspiral-merger-ringdown simulations of black-hole binaries have been performed for systems with mass ratios up to 1:10 (although most do not extend beyond 1:4), and systems with a variety of spin magnitudes and orientations, and for several choices of initial eccentricity. A periodically updated summary of simulations that include at least ten GW cycles before 
merger is given in the online version of the review [8]. Note that although a large number of simulations have been performed, these only account for an extremely sparse sampling of the full parameter space, and do not yet include any simulations of mass ratios beyond $q=m_{1} / m_{2}=10$, or of spins above $a / m \sim 0.92$. Efforts to use numerical-relativity simulations to study the astrophysics of electromagnetic counterparts have also begun $[18,19]$.

The issues for black-hole-binary simulations, as they relate to GW astronomy, are: (1) determining and achieving sufficient accuracy to extract the maximum physical information from GW observations, which includes producing simulations that include enough cycles that subsequent analytic-numerical hybrid waveforms meet these accuracy requirements, (2) producing simulations of a sufficiently dense sampling of the parameter space, (3) developing methods and codes that are efficient enough to achieve these goals.

In distinguishing the requirements on numerical waveforms between ET and other detectors, for example Advanced LIGO, the key difference is that since ET is expected to be roughly an order of magnitude more sensitive than Advanced LIGO, the waveform accuracy will also need to be higher to allow the most science to be extracted from observations. The question for numerical relativity then is what those accuracy requirements are, and whether they can be achieved before ET is completed.

\subsection{Waveform accuracy}

In the last few years questions of waveform accuracy requirements have begun to be addressed [20], and it seems that current methods and simulations are sufficiently accurate for the current generation of ground-based detectors. This statement requires some qualification and explanation. A systematic study of the accuracy of published waveforms for use in GW searches has been performed only for the case of equal-mass nonspinning binaries [21], and by necessity that study considered only binaries with high masses (so that the numerical waveforms covered the entire detector sensitivity band), and only the leading (quadrupole) harmonic. Within these limitations, however, the study showed that it will not be possible to use GW observations to distinguish between the most accurate numerical waveforms used in the study unless the signal-to-noise ratio (SNR) is above about 25 .

SNRs of that magnitude are expected to be rare from the Initial and Enhanced LIGO and Virgo detectors. However, the Einstein Telescope is expected to be an order of magnitude more sensitive, and SNRs in the range 100-1000 may be typical. We first consider whether numerical simulations will be able to match the accuracy requirements of ET by the time of its construction.

Waveform accuracy requirements are discussed in some detail in [20]. One simple criterion for waveforms to be sufficiently accurate for all parameter estimation purposes is that the average error in the amplitude and phase (appropriately weighted by the power spectrum of the detector noise [20]) 
satisfy

$$
\overline{\delta \phi}^{2}+\overline{\delta \chi}^{2}<\frac{1}{\rho^{2}}
$$

where $\rho$ is the SNR, and $\overline{\delta \chi}$ is the fractional error in the amplitude and $\overline{\delta \phi}$ is the error in the phase. If we assume that most black-hole-binary signals of interest for ET will have an SNR of less than 1000, then we require that the average phase and fractional amplitude errors be of the order of $10^{-3}$. For the waveforms described in [22] (as an example of a state-of-the-art simulation), the maximum phase and amplitude errors are roughly an order of magnitude larger than the average errors; we expect that the detector-noise-weighted average errors will behave similarly.

As such, the most accurate numerical simulations to date [23] have total phase and amplitude errors of 0.02 radian and $0.3 \%$ respectively (see the table in [21] for a convenient comparison of errors between codes), and so we expect that the average errors are already within the accuracy requirements of ET. The simulation that deals most accurately with the wave extraction [22] demonstrates errors due only to wave extraction that are also within the approximate $10^{-3}$ requirement provided here.

So it appears that state-of-the-art numerical simulations are already at, or close to, the accuracy requirements for ET science. Assuming that Moore's Law holds, then the increase in computing power by the time ET is operational will be $2^{10 / 1.5} \approx 100$. Current codes typically employ spatial derivatives that converge to fourth-order or better with respect to the spatial resolution, and if we assume fourth-order convergence in future numerical results, we will see an improvement of two orders of magnitude over current results in the next decade. This is a conservative estimate (it assumes that only the computers, and not the codes, will improve!), and serves only to demonstrate that accuracy of numerical methods are not expected to be the bottleneck in providing waveforms for ET science.

(It is true that this simple analysis does not take into account higher modes, for which comparable accuracy is harder to obtain, but for which high accuracy will be needed for parameter estimation [24-27]. However, even the subdominant modes are well-resolved in the most accurate recent simulations [28,29].)

As we will see in the next sections, the greater challenge is in sampling the black-hole-binary parameter space, and replicating the currently achievable accuracy in the simple case of equal-mass nonspinning binaries for all other binary configurations.

\subsection{Phenomenological modelling of black-hole-binary waveforms}

Individual black-hole-binary simulations can only provide a discrete sampling of the parameter space. The practical construction of template banks for searches, and the use of theoretical waveforms for parameter estimation and other follow-up studies, requires waveforms for any values of the black-hole parameters. As such, the natural approach is to construct an analytic model 
of the waveforms, and to use the numerically generated waveforms as input to calibrate the free parameters of the model. Such models also aim to extend the numerical waveforms to an arbitrarily large number of GW cycles before merger (or, put another way, extend them to arbitrarily low GW frequency).

To date there have been two approaches to this problem. One is to start with an effective-one-body (EOB) model of inspiral-merger-ringdown waveforms (which in some forms pre-date the existence of full numerical waveforms [30-34]), and to both calibrate unknown EOB coefficients to numerical results, and to introduce extra "flexibility parameters" that allow yet greater fidelity between the EOB model and the full GR results. EOB-based models for nonspinning waveforms have been pursued by several groups [35-42], and have reached an impressive level of faithfulness to the full GR waveforms [38, 41,42]. A first EOB-NR model for spinning binaries (where the black-hole spins do not precess) has also recently been presented [43].

The second approach has been to construct a purely phenomenological ansatz motivated by post-Newtonian (PN) predictions for the inspiral, approximate models of the ringdown, and the observed behaviour of the signal in the intermediate plunge and merger phase, as seen for example in [44]. This model, in which each ingredient is motivated by known physics, is extremely flexible, and has been extended from original work on nonspinning binaries [45-47] to binaries with nonprecessing spins [48] and has been shown to also be applicable to neutron-star binaries [49]. This procedure is limited only by available numerical simulations, although it remains to be seen how easily a "simple" ansatz can be developed for general precessing-spin binaries. The nonspinning waveform model is constructed from ten mass-ratio-dependent parameters, which can in turn be modelled by second-order polynomials, therefore requiring at least three simulations to define the model. The EOB models, by contrast, can be calibrated by only one simulation. It remains to be seen how well this economy of calibration extends to the spinning-binary cases, and additional simulations are necessary to validate the predictions of the model.

Both the EOB and pure phenomenological approaches require that the waveform model for the $\mathrm{GW}$ cycles before the beginning of the NR waveform (i.e., the PN or EOB model) is accurate. This requires comparison of NR waveforms with their PN counterparts in the regions where they overlap. NR-PN comparisons have been performed for equal-mass nonspinning binaries [50-53], equal-mass binaries with non-precessing spins [54], eccentric binaries [55], and one configuration of an unequal-mass binary with precessing spins [13]. These results suggest that PN methods perform sufficiently well at high frequencies (i.e., a few orbits away from merger), in the sense that most of the inspiral cycles of a numerical simulation could be replaced by PN or EOB cycles without appreciable loss from the point of view GW detection. Once again, these statements await more quantitative studies; the most precise statement that can be made at present, is that over the ten GW cycles up to about two orbits before merger, the best-performing PN approximants agree with NR simulations to within one radian in phase, and a few percent in amplitude. 
Given a range of frequencies over which both $\mathrm{PN}$ or EOB and NR results are reliable, we can then combine them to produce NR-PN hybrids. The practical question for GW astronomy, then, is how physically accurate is this "complete" waveform. This question is difficult to answer, because the PN expansion does not show good convergence properties, and it is not possible to quantify the accuracy of PN approximants over the hundreds or thousands of cycles up to the point where NR results exist. The best we can do is compare a set of different but all (hopefully) accurate PN approximants, and see how well they agree. First studies of the accuracy of equal-mass hybrids at least suggest that they will fulfil the detection requirements of current ground-based detectors for masses as low as $10 M_{\odot}$ [56]. Complementary results for studies of pure PN inspiral waveforms suggest that they in turn will be sufficient for masses up to $12 M_{\odot}$ [57]. However, the wider sensitivity band of ET may require that the hybrid waveforms include more accurate PN ingredients, or longer numerical simulations. In contrast to the physical and computational accuracy of the raw numerical waveforms, the question of how long these waveforms must be, and how well their PN counterparts perform at low frequencies, is not so clear. These are topics that will be an important focus of research in the next few years.

\subsection{Sampling of the parameter space}

Whichever phenomenological approach turns out to be most practical (and indeed the pursuit of multiple approaches appears at present to be the wisest course), it is clear that simulations covering a fine sampling of the black-holebinary parameter space will be necessary. An exhaustive sampling of the parameter space is usually presented as a monumental computational challenge. Let us consider the scope of this challenge, and the degree to which it may be met by the time ET is commissioned.

There are nine parameters in the black-hole-binary parameter space. A recent phenomenological family of inspiral-merger-ringdown waveforms [48] was able to faithfully model binaries with non-precessing spins using an analytic ansatz in which the coefficients were expressed as third-order polynomials in the physical parameters of the model (in this case the mass ratio and a parameter that can be approximately considered as the total spin of the binary). For simplicity, let us take the relatively optimistic view that third-order polynomials in each of the parameters will be sufficient to model the full parameter space, and that we therefore require at least four data points in each direction to robustly constrain our model. This means that we will require $4^{8} \approx 65,000$ simulations. Considering that typical simulations can require on the order of tens (and in some cases hundreds) of thousands of CPU hours each, this would indeed be a monumental undertaking!

However, this does not take into account symmetries and degeneracies in the parameter space. For example, the work in [58] successfully parameterizes non-precessing binaries by only the total spin of the binary — with the 
consequence that if the individual spins aren't necessary to model the waveform, then they will also be difficult to distinguish. This reduces the amount of information that can be obtained from a detection based on this waveform model, but also reduces the computational cost of producing the model.

As a second example, the works of $[59,60]$ exploit the symmetries of blackhole-binary systems to argue that certain quantities in black-hole mergers (the mass, spin and recoil velocity of the final black hole) can be described by a model that requires only 42 simulations to calibrate. Their model deals with only quasi-circular inspiral (so the parameter space is reduced to seven, not nine, parameters), and uses a second-order-polynomial ansatz, but nonetheless requires far fewer simulations than a naive estimation of $3^{7} \approx 2200$.

These early attempts to characterize black-hole-binary systems suggest that a full mapping of the parameter space may be possible within the next decade, if not the next few years. Before becoming overly optimistic, however, we should bear in mind that the error analyses of many current simulations deal only with the dominant harmonic (the $\ell=2, m=2$ mode) of the waveform, and to date it is only this mode that has been included in analytic/phenomenological models of black-hole-binary waveforms. Also, two important areas of the parameter space have not yet been accessed by current simulations.

One is black holes with high spin. All published long simulations to date have used black-hole initial data that are conformally flat, an assumption that simplifies the construction of the data, but limits the spin that can be modeled to about $a / m<0.92$, although accurate binary simulations have been performed close to that limit [61]. The behaviour of black-hole-binary systems with near-extremal spins (which may be the most common astrophysically [6264]), have therefore not yet been studied in detail. Proposals have been made to construct non-conformally-flat initial data for both excision-based $[65,66]$ and moving-puncture [67] codes, although only the excision data is sufficiently developed for use in full binary simulations. With workable proposals in place for high-spin data, it is likely that high-spin binaries will be simulated in detail in the next few years, and this region of the parameter space will be well modelled by the time of ET.

The situation is different for large mass ratios. In current simulations, the numerical resolution requirements of the simulation are defined by the smallest black hole, while the overall scale is set by the total mass. This means that the higher the mass ratio $\left(q=m_{1} / m_{2} \geq 1\right)$, the more effective resolution is required, and the more computationally expensive the simulation. Long, accurate simulations suitable for use in GW applications have been performed up to $q=6$ [39], and a short simulation of $\approx 2.5$ orbits [68] exists for $q=10$. Since the simulation cost (both in simulation time and memory usage) scales at best linearly with the mass ratio, and more realistically quadratically, long accurate simulations of mass ratios $q \sim 10$ are at the very limit of current methods and resources, and $q \sim 100$ are out of the question. An improvement by a factor of 100 in computer power by the time of ET suggests that mass 
ratios $q \sim 30$ may be possible by that time, but $q \sim 100$ will require new techniques; some possibilities are described in Section 2.4.

It may ultimately not be necessary to simulate systems with $q>10$ in full general relativity to accurately model the full parameter space. For example, the work in [48] includes simulations up to only $q=3$, but models all mass ratios by explicitly incorporating an analytically calculated extreme-mass-ratio limit. The effective-one-body (EOB) procedure also includes by construction the extreme-mass-ratio limit [30]. However, in order to verify that the high mass ratios are indeed being faithfully modelled, it will be necessary to perform at least a few simulations at very high mass ratios. Even if only a few such simulations are required to verify the fidelity of a given model (or set of models), new techniques will be necessary to produce those simulations.

\subsection{Numerical techniques and room for improvement}

The accuracy and length of black-hole-binary waveforms has improved significantly since the first simulations of 2005. The first waveforms covered only a few GW cycles before merger, while the most recent simulations cover up to 30 cycles before merger. The first long simulations that allowed comparison with post-Newtonian (PN) results had an amplitude uncertainty of around $10 \%$ and an accumulated phase error of around 1 radian before merger [50]. The most recent simulations can achieve an amplitude error of $0.3 \%$ and an accumulated phase error through inspiral, merger and ringdown of $0.02 \mathrm{rad}$ [28].

Typically mesh-refinement is used to resolve both the small-scale features near the black holes, the medium-scale physics of the GW signal, and the large scales necessary to push the outer boundary of the computational domain far from the source. However, improvements in general code efficiency and the introduction of higher-order spatial finite-differencing (first sixth-order in [69, 51], and later eighth-order in [70,13]) have allowed impressive improvements in both code speed, memory efficiency, and numerical accuracy. More recent results by two groups $[71,29]$ have demonstrated the efficacy of multipatch methods, which allow for a far more efficient gridding of the wave extraction region, which may lead to yet greater accuracy (of the wave extraction) and computational efficiency in future simulations. The results of Reisswig et. al. have further achieved Cauchy-Characteristic Extraction (CCE), in which the waves extracted at some finite spatial distance from the source are then evolved to null infinity, which is where GW signals can be unambiguously defined [22, 72]. These results indeed suggest that this method produces results that are gauge invariant.

Simulations with variants of the generalized-harmonic formulation have been performed by Pretorius [73,5,74] and by the Caltech-Cornell group [75, 53]. The latter's SpEC code makes use of a multi-domain spectral method, which allows a high degree of accuracy and, like the multi-patch methods described above, allow high accuracy with minimal computational resources in the wave extraction region. (Note that the terms "multi-domain" and "multi- 
patch" refer to the same idea, although the implementations of this idea in the two codes we have referred to are quite different. Our choice of labels only reflects the way the codes' authors refer to them in the literature.)

These codes represent the current state of the art. However, far greater accuracy and efficiency will be needed to meet the science goals of ET. Is there room for improvement?

The answer is certainly Yes. A number of options are yet to be fully explored. One that has already received some attention is the use of mixed implicit-explicit (IMEX) time integration to allow far larger evolution time steps. A first exploration of this approach has already been made in the context of scalar fields on a curved background [76], but motivated by, and using a code developed for, black-hole-binary simulations. Another approach with a related potential benefit is the use of symplectic integrators [77-80]. In this approach, conserved quantities in the physical system are maintained by construction, and this in general allows for far larger time steps. The canonical example is the evolution of binaries in Newtonian gravity, where dramatic improvements in accuracy are possible. General relativistic systems are of course far more complex, and there is the added difficulty that energy can be dissipated as gravitational radiation, but these issues do not preclude the use of symplectic methods, and some research on this topic is already underway.

A further improvement in both accuracy and memory efficiency could be achieved by the use of hyperboloidal slices of the spacetime. Here the slices are spacelike near the source, but asymptotically null, meaning that they reach null infinity on a finite numerical domain. Since the GW phase is constant on a null slice, only a finite number of GW cycles need be resolved between the source and the outer boundary. This approach would therefore save computational resources in the wave zone, while at the same time allowing clean GW extraction at null infinity. Although hyperboloidal initial data for such systems has already been proposed $[81,82]$, stable simulations are more challenging. Progress has however been made in treating perturbations on Schwarzschild and Kerr backgrounds [83,84], and in evolving single-black-hole spacetimes in spherical symmetry and axisymmetry $[85,86]$, and hyperboloidal simulations of black-hole binaries may be possible in the next few years.

In the case of moving-puncture simulations, improvements may also be possible by better exploiting the natural geometry of black-hole slices in this approach. The method was developed for data that describe the black holes as topological wormholes, but it was later found that the slices quickly asymptote to cylinders that end at the black-hole throat (also called "trumpets") [8789]. It may be possible to modify the method to exploit more explicitly this trumpet form of the data. In particular, if the original concept of the puncture approach can be resurrected - where the singular nature of the data near the black holes is described analytically and only small deviations from this form (due to the presence of the second black hole) are described numerically then it may be possible to achieve dramatic improvements in code accuracy and efficiency. Related ideas have also been suggested for dealing with extrememass-ratio binaries [90]. 
These are a number of avenues for improvement that are already being explored, and have the potential by themselves to make possible large numbers of long, accurate simulations possible - but of course we expect that by 2020 there will be many other ideas, and it is quite conceivable that by then the standard methods of the field will be radically different from those today.

\section{Matter}

In contrast to simulations of binary black holes, many calculations of gravitational waves have not used full general relativity (for example most calculations of core-collapse as reviewed by e.g. [91] and some binary simulations such as [92]). This is unsurprising; for scenarios such as core-collapse supernovae the effect of including the key physics in the model is more important than a perfect model of gravity. However, with the recent successes in evolving the spacetime as detailed in section 2 the use of full general relativity in calculations of gravitational waves is likely to become standard on the timescales important for ET.

In addition we note that as the matter terms do not affect the principal part of the spacetime evolution equations there is only one potential complication for the spacetime evolution by adding matter. In vacuum evolutions, away from singularities, all quantities remain smooth. As the matter quantities may become discontinuous, a simulation that includes such shocks will have an effect on the spacetime. It is currently unclear what the impact is in GW simulations, as the only work to look at this ([93]) is in $1+1$ dimensions. As no effect has been seen in any current simulation we will assume that no fundamental problem will affect matter simulations relevant for ET.

From these basic points we will assume that all current GW simulations including matter will be able to benefit from the theoretical and numerical advances for spacetime and gauge evolution and wave extraction reviewed or proposed in section 2 . Therefore the bottleneck in computing accurate, realistic GW signals will be in the physical model of the matter that can practically be evolved, and the accuracy of the numerical methods used to evolve it.

The parameter space for matter simulations is considerably larger than for binary black holes. Firstly the underlying physics of the problem sets the mass scale which cannot be removed. Secondly there are a wide variety of GW sources; as well as binary systems there are discs and single star collapse. But most importantly there is the uncertainty in the physics that the model must include to be realistic (e.g., neutrino transport plays a crucial role in core collapse but is much less important in a binary inspiral), and in the details of that physics (such as the precise form of the equation of state (EOS) needed to close the perfect fluid equations).

The approach so far has been to focus on limited areas in the parameter space to determine which elements of the physical model affect the qualitative form of the GWs, and if the GW signal is relatively robust to determine what qualitative effects minor changes in the parameters make. The key additional 
parameters in inspirals are currently believed to be the bulk EOS and the magnetic field; in core collapse neutrinos and composition are more important than magnetic fields. Additional effects such as superfluidity, an elastic crust, diffusion, dissipation, or other non-ideal effects may play a role. At present even the simplest implementation of these effects is ongoing research.

The construction of initial data for binary systems is similar to that for binary black holes and, as reviewed by $[15,10]$, similar issues of the accuracy of the conformal flatness approximation and how to produce quasi-equilibrium initial data arise. For single star collapse either axi-stationary solutions are perturbed or a pre-calculated model is used, with similar concerns about the physical accuracy of the initial conditions. In most simulations small parameter studies show that the qualitative behaviour of the gravitational waves is robust; one fundamental problem will be discussed later.

The key question for GWs and in particular for ET is whether numerical relativity can compute sufficiently accurate signals. As this crucially depends on the physics included in the model to be simulated we will discuss the different input physics separately.

\subsection{Hydrodynamics}

Due to the scale and temperature of most interesting GW sources the bulk of the matter can be reasonably modelled as a fluid, and in highly dynamical situations such as a binary merger the bulk ideal fluid effects, modelled by the relativistic Euler equations, will dominate. These equations are typically written in conservation law form [94] as they admit discontinuous (weak) solutions for which the conservation law form gives a unique entropy satisfying solution. The microphysics under consideration is determined by the EOS which closes the system of equations and is typically given as a relation between the pressure and the independent thermodynamic and composition variables.

The importance of the EOS is obvious, as has been illustrated by a number of simulations (as reviewed by [10], but see in particular $[92,95,96])$. Large and small scale effects both in terms of the stiffness and the effects of heat in simplified form have been covered. However current uncertainty in the EOS is large, and at present the approaches followed are to restrict to the "best" EOS currently available or to use a simple parametrized EOS ([97]) to best fit the likely candidates. The effectiveness of the parametrized EOS combined with numerical simulations has been illustrated by [98], although it should be noted that the accuracy of the simulations discussed there will not be sufficient for ET.

Numerical solutions of the relativistic Euler equations use techniques from the standard Newtonian Euler equations. In particular it is currently typical to use High Resolution Shock Capturing (HRSC) methods (see e.g. [99,100] and references therein for reviews). These ensure conservation is preserved (necessary to ensure the correct weak solution is obtained if a shock is present) and ensure that the solution converges near discontinuities. These techniques 
are nonlinear in the sense that the information used at any given point depends on the data in the neighbourhood of that point; this is required to bypass the conditions of Godunov's theorem ([101]) and construct numerical methods that give correct solutions that converge faster than first order.

The techniques currently used in simulations are extremely similar, even when the spacetime is evolved using spectral methods $([102,103])$, multiple patches ([104]) or a mesh refined cartesian grid (e.g. [105-107]). In nearly every case a finite volume approach combined with a reconstruction-evolution method and approximate Riemann solvers is used, computing a conservative update over the neutron star and the surrounding artificial atmosphere. These methods converge at best at second order and are computationally expensive compared to the methods required for the spacetime.

These methods have been used to study purely hydrodynamical scenarios. The numerical accuracy of the resulting GW signals is not always easy to assess, with some work at lower resolutions not claiming accuracy of better than 1 orbit ([98]), whilst others at the highest currently available resolutions claiming accuracy in key quantities of the order of a percent $([108])$. Even in simulations with careful control of the accuracy in the signal the amplitude accuracy is nearly an order of magnitude worse than early black hole binary simulations (compare figure 5 of [108] with figure 3 of [109]). It is also clear that numerical accuracy is significantly degraded when shocks appear such as in the complex post-merger stage of a binary inspiral. Thus whilst it seems that current techniques and computing power are sufficient to make qualitative statements about gravitational waves and the general effects of changes in the parameter space, detailed quantitative predictions at the level required by ET are unlikely to result simply by throwing computing power at current techniques, except in certain simple cases.

Improvements in numerical techniques to significantly improve the accuracy of the results, particularly in the complex post-merger regime, are therefore needed to get the most out of numerical relativity for ET. Extensions of standard finite volume techniques to higher order (e.g. [110]) are one obvious avenue to explore. The implementation of more complex but flexible methods such as Discontinuous Galerkin finite elements (as implemented in relativity by $[111,112])$ is another possibility. Finite difference methods have received some attention (e.g. $[113,114])$ and may be the simplest approach. Another possibility is the use of smoothed-particle hydrodynamics which has been employed in NS simulations in approximations to GR by e.g. $[92,115,116]$. Recent results such as [117] suggest that consistent implementations using modern techniques that avoid problems near shocks may soon appear, but their numerical accuracy will need careful assessment. At present it seems unlikely that the highly accurate spectral methods will provide a solution due to the problems these methods encounter with discontinuities (although [118] presents one approach in a particular approximation), but hybrid methods (e.g. $[119,120]$ ) may allow for a combination of the best features of spectral or simple high order schemes with HRSC methods. 
Finally it should be noted that the impact of the artificial atmosphere used outside the compact GW source on the accuracy of the results remains unclear, and methods to avoid the use of an atmosphere (e.g. [121,122]) are at present not sufficiently advantageous to use in relevant simulations. The presence of a free surface (in the perfect fluid model) may be an impediment to the use of more accurate numerical methods, and remains an area where more understanding at both numerical and theoretical level is needed. Purely on numerical grounds the use of finite elements is attractive here, as the grid can be adapted to the surface of the objects; all that is required is a consistent boundary condition to impose.

\subsection{Magnetic fields}

So far the ideal MHD limit has been the focus of a range of studies (for a recent review see [10]), many simply "adding on" the magnetic field to study the qualitative differences (see e.g. $[123,124]$ ), but some studying additional instabilities that may arise (e.g. [116,125]). With the increase in the parameter space due to the strength and topology of the magnetic field the choice of initial conditions becomes ever more important, and this is where one fundamental difficulty remains. It is expected that the initial object will be approximately axisymmetric and stationary (axi-stationary) and the precise topology and mixture of toroidal and poloidal components that would make up this initial field has only been studied numerically under certain assumptions, see [126128]. Until a self-consistent quasi-stationary solution can be constructed the standard method is to add a purely poloidal magnetic field to the interior; it has been argued ([123]) that this is sufficient for the inspiral phase of a magnetized binary.

In addition to the conservation constraints, numerical methods should identically preserve the $\nabla \cdot \mathbf{B}=0$ constraint. There are many possible approaches, with most codes implementing a variant of constrained transport (e.g. [129]) and some using hyperbolic divergence cleaning (e.g. [114]). Constrained transport methods should ensure the constraint is satisfied to machine precision whilst divergence cleaning propagates any errors away; however divergence cleaning is simpler to extend to higher order methods and the presence of the neutron star surface and artificial atmosphere may affect the accuracy of constrained transport. In all cases the additional constraints to be satisfied and fields to be evolved lead to lower numerical accuracy; for example [123] shows the numerical error increasing by factors $\sim 5$ for evolutions of magnetic binaries compared to non-magnetic NSs.

\subsection{Additional effects}

In the context of computing GWs for ET the importance of physics beyond the ideal MHD model are the changes to the internal structure, which will 
certainly affect the fine detail and can change the dynamics, leading to a qualitative change in the signal. The most obvious problems arise through thermal effects (radiation transport and diffusion post-merger or in core-collapse) or where the ideal fluid model breaks down (viscous or multifluid effects such as superfluidity, and in the crust).

\subsubsection{Radiation transport}

The transfer of heat and energy through radiation transport changes the local structure of the fluid, and is known to be crucial in the dynamics of corecollapse supernovae and may play a role in the post-merger dynamics of a binary merger. However the simulation of the full Boltzmann equation in $3+$ $3+1$ dimensional general relativity would seem to be impractical on timescales relevant for ET. An estimate ([130]) suggests that even approximate transport methods require peta scale computing power. A suggestion for modelling the full problem is given by [131], but on the timescale for ET it seems likely that approximation methods such as [132] will be the only practical solution.

\subsubsection{Non-ideal effects}

The consideration of non-ideal effects may become important post-merger when the matter surrounding the remnant can be both very hot and yet not too dense, leading to a plasma where diffusion and resistivity are important. Viscous effects have barely been touched on - for an isolated example see [133]. Resistivity effects have been considered and modelled in the magnetosphere, but modelling for neutron stars is only just starting (see $[111,112]$ ).

The Newtonian limit gives mixed hyperbolic-parabolic systems of equations with their associated causality problems. The relativistic equivalents have stiff source terms leading to severe timestep constraints when using standard numerical methods. Various numerical schemes have been proposed to bypass this issue, including the use of IMEX time integrators ([111]). It seems plausible that similar numerical techniques will be adopted as those for large mass ratio binary black holes.

\subsubsection{Multifluid and elastic effects}

It is believed that most neutron stars have a region containing superfluid neutron pairs and that magnetized neutron stars may be superconducting in some region. The crust of a mature neutron star is not a fluid but a lattice, best modelled as a relativistic elastic solid inter-penetrated by an additional fluid. In addition, the possible existence of exotic phases of matter in the inner core leads to another region where multiple particles may be flowing freely and independently. All of these effects involve the local interaction of multiple fluids or solids (for a review see [134]), with the appearance of additional dynamics and instabilities. 
At present no numerical evolutions of any of these effects have been performed. Frameworks for evolving multiple fluids have been worked out ([134]) but have not been tailored for numerical work that would include shocks. It seems unlikely that multifluid effects will be visible in numerical simulations in the inspiral phase, but the additional propagation speeds and instabilities could be visible near or post merger.

\section{Discussion: the nature of numerical relativity in the ET era}

Over the last four decades, numerical relativists have been primarily concerned with the technical challenges of solving Einstein's equations numerically: formulating Einstein's equations to be numerically stable, developing appropriate gauge conditions, exploring the numerical techniques necessary to accurately evolve relativistic spacetimes, and to handle relativistic fluids and magnetic fields. Many of these issues have been resolved to a sufficient degree that we can now extract useful physics from the simulations. In addition, sensitive GW detectors are now in operation and the analysis of their data requires numerical results. The field of numerical relativity is thus shifting from a focus on theoretical and numerical-analysis issues to questions of astrophysics and GW data analysis.

Over the next decade, this shift will continue, and numerical relativists will interact more closely with astronomers, astrophysicists, and GW data analysts. Closer collaboration between the NR and DA communities has already begun through the Numerical INJection Analysis (NINJA) project [135], where numerical black-hole-binary waveforms were injected into simulated Initial LIGO and Virgo detector noise, and then the data were analyzed by a battery of search and parameter estimation methods, to test their efficacy in dealing with "real" GW signals. The successful first NINJA project [136] is now being followed with projects to more systematically test search pipelines. Among these are the suggestion to use actual detector data, which contain the full array of real detector artifacts and can allow the most conclusive tests of search pipelines, and to extend the original simulated-detector-noise NINJA strategy to matter sources (NS-NS, NS-BH and core collapse) [137].

With the advent of Advanced LIGO/Virgo and the space-based detector LISA [138], the interaction between the NR and DA communities will surely become closer, and the possibility of multi-messenger GW astronomy will include astronomers and astrophysicists in these efforts, too. Once GW detections become routine, and numerical simulations computationally cheaper and faster, it may be typical for a candidate observation to motivate follow-up numerical simulations, which in turn lead to more sophisticated data analysis parameter estimation exercises, and comparison with electromagnetic observations. The time scale of this back-and-forth interaction may be as short as weeks or even days. For some regions of the (at least black-hole-binary) parameter space, it is in fact likely that GW astronomers will have ready access to numerical codes that allow them to "dial up" any waveform they require 
for a particular data-analysis exercise, and the focus of numerical-relativity research will be only on those cases (for example, high mass ratios, binaries with complex spin interactions, and matter systems) that still present problems.

For matter simulations considerable work has been done to explore the parameter space by including as much of the relevant physics as is currently practical. However, in order to take full advantage of the additional accuracy given by ET these simulations will have to improve substantially in certain areas. Firstly the numerical methods currently employed will probably not deliver the improvements in accuracy required based on the (conservative) increase in computing power projected here. Secondly the community will have to collaborate closely with the data analysis community in order to determine which aspects of the physical model will actually lead to interesting observable effects, and hence where in the parameter space the numerical simulations should be focused. As the discussion in Section 3 makes clear it would be possible to spend all the time and increased computing power in applying and simulating better physical models and exploring the parameter space. It seems likely that to take the greatest advantage of ET a narrower focus is needed to produce the most accurate simulations including the most relevant physics for the best candidate sources.

Acknowledgements The authors thank John Baker, Manuel Tiglio and Anil Zenginoglu for helpful comments on the manuscript. MH was supported by SFI grant 07/RFP/PHYF148 at University College Cork, and by FWF Lise-Meitner project M1178-N16 at the University of Vienna; and thanks the University of the Balearic Islands for hospitality while some of this work was completed, and Sascha Husa for numerous discussions. IH was supported by the STFC rolling grant PP/E001025/1 and Nuffield Foundation grant NAL/32622.

\section{References}

1. B. Abbott, et al., (2007). arXiv:0711.3041

2. F. Acernese, et al., Class. Quantum Grav. 23, S635 (2006)

3. S. Hild (for the LIGO Scientific Collaboration), Class. Quantum Grav. 23, S643 (2006)

4. B.S. Sathyaprakash, B.F. Schutz, Living Rev. Rel. 12, 2 (2009)

5. F. Pretorius, Phys. Rev. Lett. 95, 121101 (2005). DOI 10.1103/PhysRevLett.95.121101

6. M. Campanelli, C.O. Lousto, P. Marronetti, Y. Zlochower, Phys. Rev. Lett. 96, 111101 (2006)

7. J.G. Baker, J. Centrella, D.I. Choi, M. Koppitz, J. van Meter, Phys. Rev. Lett. 96, 111102 (2006)

8. M. Hannam, Class. Quant. Grav. 26, 114001 (2009). DOI 10.1088/0264$9381 / 26 / 11 / 114001$

9. M. Shibata, K. Uryu, Phys. Rev. D61, 064001 (2000). DOI 10.1103/PhysRevD.61.064001

10. J. Faber, Classical and Quantum Gravity 26(11), 114004 (18pp) (2009). URL http://stacks.iop.org/0264-9381/26/114004

11. H.P. Pfeiffer, et al., Class. Quant. Grav. 24, S59 (2007). DOI 10.1088/02649381/24/12/S06

12. S. Husa, M. Hannam, J.A. Gonzalez, U. Sperhake, B. Brügmann, Phys. Rev. D77, 044037 (2008). DOI 10.1103/PhysRevD.77.044037

13. M. Campanelli, C.O. Lousto, H. Nakano, Y. Zlochower, Phys. Rev. D79, 084010 (2009)

14. M. Alcubierre, Introduction to $3+1$ Numerical Relativity (Oxford University Press, USA, 2008) 
15. G.B. Cook, Living Rev. Rel. 3, 5 (2000)

16. F. Pretorius, in Physics of relativistic objects in compact binaries: from birth to coalescence, vol. 359, ed. by M. Colpi, P. Casella, V. Gorini, U. Moschella, A. Possenti (Springer-Verlag, 2009), vol. 359, pp. 269-305. arXiv:0710.1338

17. S. Husa, Eur. Phys. J. ST 152, 183 (2007)

18. C. Palenzuela, M. Anderson, L. Lehner, S.L. Liebling, D. Neilsen, (2009). arXiv:0905.1121

19. J.R. van Meter, et al., (2009). arXiv:0908.0023

20. L. Lindblom, B.J. Owen, D.A. Brown, Phys. Rev. D78, 124020 (2008). DOI 10.1103/PhysRevD.78.124020

21. M. Hannam, et al., Phys. Rev. D79, 084025 (2009)

22. C. Reisswig, N.T. Bishop, D. Pollney, B. Szilagyi, (2009). arXiv:0907.2637

23. M.A. Scheel, et al., Phys. Rev. D79, 024003 (2009). DOI 10.1103/PhysRevD.79.024003

24. A.M. Sintes, A. Vecchio, (1999). gr-qc/0005058

25. C. Van Den Broeck, A.S. Sengupta, Class. Quant. Grav. 24, 1089 (2007). DOI 10.1088/0264-9381/24/5/005

26. S. Babak, M. Hannam, S. Husa, B. Schutz, (2008). arXiv:0806.1591

27. J.I. Thorpe, et al., (2008). arXiv:0811.0833

28. M. Boyle, et al., Phys. Rev. D78, 104020 (2008). DOI 10.1103/PhysRevD.78.104020

29. D. Pollney, Talk given at NRDA2009, AEI-Potsdam, July 6-9, 2009

30. A. Buonanno, T. Damour, Phys. Rev. D 59, 084006 (1999)

31. A. Buonanno, T. Damour, Phys. Rev. D 62, 064015 (2000)

32. T. Damour, P. Jaranowski, G. Schäfer, Phys. Rev. D 62, 084011 (2000)

33. T. Damour, Phys. Rev. D 64, 124013 (2001)

34. A. Buonanno, Y. Chen, T. Damour, Phys. Rev. D74, 104005 (2006)

35. A. Buonanno, et al., Phys. Rev. D76, 104049 (2007)

36. T. Damour, A. Nagar, Phys. Rev. D77, 024043 (2008). DOI 10.1103/PhysRevD.77.024043

37. T. Damour, A. Nagar, E.N. Dorband, D. Pollney, L. Rezzolla, Phys. Rev. D77, 084017 (2008). DOI 10.1103/PhysRevD.77.084017

38. T. Damour, A. Nagar, M. Hannam, S. Husa, B. Brügmann, Phys. Rev. D78, 044039 (2008). DOI 10.1103/PhysRevD.78.044039

39. J.G. Baker, et al., Phys. Rev. D78, 044046 (2008). DOI 10.1103/PhysRevD.78.044046

40. A.H. Mroue, L.E. Kidder, S.A. Teukolsky, Phys. Rev. D78, 044004 (2008). DOI 10.1103/PhysRevD.78.044004

41. T. Damour, A. Nagar, (2009). arXiv:0902.0136

42. A. Buonanno, et al., (2009). arXiv:0902.0790

43. Y. Pan, et al., (2009)

44. A. Buonanno, G.B. Cook, F. Pretorius, Phys. Rev. D75, 124018 (2007). DOI 10.1103/PhysRevD.75.124018

45. P. Ajith, et al., Class. Quant. Grav. 24, S689 (2007). DOI 10.1088/02649381/24/19/S31

46. P. Ajith, et al., Phys. Rev. D77, 104017 (2008). DOI 10.1103/PhysRevD.77.104017

47. P. Ajith, Class. Quant. Grav. 25, 114033 (2008). DOI 10.1088/0264$9381 / 25 / 11 / 114033$

48. P. Ajith, et al., (2009)

49. M. Shibata, K. Kyutoku, T. Yamamoto, K. Taniguchi, Phys. Rev. D79, 044030 (2009). DOI 10.1103/PhysRevD.79.044030

50. J.G. Baker, J.R. van Meter, S.T. McWilliams, J. Centrella, B.J. Kelly, Phys. Rev. Lett. 99, 181101 (2007). DOI 10.1103/PhysRevLett.99.181101

51. M. Hannam, S. Husa, U. Sperhake, B. Brügmann, J.A. Gonzalez, Phys. Rev. D77, 044020 (2008). DOI 10.1103/PhysRevD.77.044020

52. A. Gopakumar, M. Hannam, S. Husa, B. Bruegmann, Phys. Rev. D78, 064026 (2008). DOI 10.1103/PhysRevD.78.064026

53. M. Boyle, et al., Phys. Rev. D76, 124038 (2007). DOI 10.1103/PhysRevD.76.124038

54. M. Hannam, S. Husa, B. Bruegmann, A. Gopakumar, Phys. Rev. D78, 104007 (2008). DOI 10.1103/PhysRevD.78.104007

55. I. Hinder, F. Herrmann, P. Laguna, D. Shoemaker, (2008) 
56. M. Hannam, et al., (2009). In preparation

57. A. Buonanno, B. Iyer, E. Ochsner, Y. Pan, B.S. Sathyaprakash, (2009)

58. P. Ajith, et al., (2009). In preparation

59. L. Boyle, M. Kesden, S. Nissanke, Phys. Rev. Lett. 100, 151101 (2008). DOI 10.1103/PhysRevLett.100.151101

60. L. Boyle, M. Kesden, Phys. Rev. D78, 024017 (2008). DOI 10.1103/PhysRevD.78.024017

61. S. Dain, C.O. Lousto, Y. Zlochower, Phys. Rev. D78, 024039 (2008). DOI 10.1103/PhysRevD.78.024039

62. M. Volonteri, P. Madau, E. Quataert, M.J. Rees, Astrophys. J. 620, 69 (2005). DOI $10.1086 / 426858$

63. C.F. Gammie, S.L. Shapiro, J.C. McKinney, Astrophys. J. 602, 312 (2004)

64. S.L. Shapiro, Astrophys. J. 620, 59 (2005)

65. G. Lovelace, R. Owen, H.P. Pfeiffer, T. Chu, Phys. Rev. D78, 084017 (2008). DOI 10.1103/PhysRevD.78.084017

66. G. Lovelace, Class. Quant. Grav. 26, 114002 (2009). DOI 10.1088/0264$9381 / 26 / 11 / 114002$

67. M. Hannam, S. Husa, B. Brügmann, J.A. Gonzalez, U. Sperhake, Class. Quantum Grav. 24, S15 (2007)

68. J.A. Gonzalez, U. Sperhake, B. Brugmann, Phys. Rev. D79, 124006 (2009). DOI 10.1103/PhysRevD.79.124006

69. S. Husa, J.A. Gonzalez, M. Hannam, B. Brügmann, U. Sperhake, Class. Quant. Grav. 25, 105006 (2008). DOI 10.1088/0264-9381/25/10/105006

70. C.O. Lousto, Y. Zlochower, Phys. Rev. D77, 024034 (2008). DOI 10.1103/PhysRevD.77.024034

71. E. Pazos, M. Tiglio, M.D. Duez, L.E. Kidder, S.A. Teukolsky, (2009). arXiv:0904.0493

72. J. Winicour, Living Reviews in Relativity 12(3) (2009). URL http://www.livingreviews.org//rr-2009-3

73. F. Pretorius, Class. Quantum Grav. 22, 425 (2005)

74. F. Pretorius, Class. Quantum Grav. 23, S529 (2006)

75. L. Lindblom, M.A. Scheel, L.E. Kidder, R. Owen, O. Rinne, Class. Quantum Grav. 23, S447 (2006)

76. S.R. Lau, H.P. Pfeiffer, J.S. Hesthaven, (2008). arXiv:0808.2597

77. J.D. Brown, Phys. Rev. D73, 024001 (2006). DOI 10.1103/PhysRevD.73.024001

78. R. Richter, C. Lubich, Class. Quant. Grav. 25, 225018 (2008). DOI 10.1088/0264$9381 / 25 / 22 / 225018$

79. R. Richter, Class. Quant. Grav. 26, 145017 (2009). DOI 10.1088/0264$9381 / 26 / 14 / 145017$

80. J. Frauendiener, (2008). arXiv:0805.4465

81. F. Ohme, M. Hannam, S. Husa, N.O. Murchadha, (2009). arXiv:0905.0450

82. L.T. Buchman, H.P. Pfeiffer, J.M. Bardeen, (2009). arXiv:0907.3163

83. A. Zenginoglu, D. Nunez, S. Husa, Class. Quant. Grav. 26, 035009 (2009). DOI 10.1088/0264-9381/26/3/035009

84. A. Zenginoglu, M. Tiglio, Phys. Rev. D80, 024044 (2009). DOI 10.1103/PhysRevD.80.024044

85. V. Moncrief, O. Rinne, Class. Quant. Grav. 26, 125010 (2009). DOI 10.1088/0264$9381 / 26 / 12 / 125010$

86. O. Rinne, Class. Quant. Grav. 27, 035014 (2010). DOI 10.1088/0264$9381 / 27 / 3 / 035014$

87. M. Hannam, S. Husa, D. Pollney, B. Bruegmann, N. O'Murchadha, Phys. Rev. Lett. 99, 241102 (2007). DOI 10.1103/PhysRevLett.99.241102

88. M. Hannam, S. Husa, F. Ohme, B. Bruegmann, N. O'Murchadha, Phys. Rev. D78, 064020 (2008). DOI 10.1103/PhysRevD.78.064020

89. M. Hannam, S. Husa, N.O. Murchadha, (2009). arXiv:0908.1063

90. S. Detweiler, Talk given at 7th Lisa Symposium, Barcelona, June 16-20 2008

91. C.D. Ott, Class. Quant. Grav. 26, 063001 (2009). DOI 10.1088/0264$9381 / 26 / 6 / 063001$

92. R. Oechslin, H.T. Janka, Phys. Rev. Lett. 99, 121102 (2007). DOI 10.1103/PhysRevLett.99.121102 
93. A.P. Barnes, P.G. Lefloch, B.G. Schmidt, J.M. Stewart, Class. Quant. Grav. 21, 5043 (2004). DOI 10.1088/0264-9381/21/22/003

94. F. Banyuls, J.A. Font, J.M. Ibáñez, J.M. Martí, J.A. Miralles, Astrophys. J. 476, 221 (1997)

95. K. Kiuchi, Y. Sekiguchi, M. Shibata, K. Taniguchi, (2009). arXiv:0904.4551

96. L. Baiotti, B. Giacomazzo, L. Rezzolla, Phys. Rev. D78, 084033 (2008). DOI 10.1103/PhysRevD.78.084033

97. J.S. Read, B.D. Lackey, B.J. Owen, J.L. Friedman, Phys. Rev. D79, 124032 (2009). DOI 10.1103/PhysRevD.79.124032

98. J.S. Read, et al., Phys. Rev. D79, 124033 (2009). DOI 10.1103/PhysRevD.79.124033

99. J.A. Font, Living Reviews in Relativity 11(7) (2008). URL http://www.livingreviews.org/lrr-2008-7

100. J.M. Martí, E. Müller, Living Reviews in Relativity 6(7) (2003). URL http://www.livingreviews.org//rr-2003-7

101. S.K. Godunov, Mat. Sb. 47, 271 (1959)

102. H. Dimmelmeier, J. Novak, J.A. Font, J.M. Ibanez, E. Muller, Phys. Rev. D71, 064023 (2005). DOI 10.1103/PhysRevD.71.064023

103. M.D. Duez, et al., Phys. Rev. D78, 104015 (2008). DOI 10.1103/PhysRevD.78.104015

104. B. Zink, E. Schnetter, M. Tiglio, Phys. Rev. D77, 103015 (2008). DOI 10.1103/PhysRevD.77.103015

105. L. Baiotti, I. Hawke, L. Rezzolla, E. Schnetter, Phys. Rev. Lett. 94, 131101 (2005). DOI 10.1103/PhysRevLett.94.131101

106. T. Yamamoto, M. Shibata, K. Taniguchi, Phys. Rev. D78, 064054 (2008). DOI 10.1103/PhysRevD.78.064054

107. M. Anderson, et al., Phys. Rev. Lett. 100, 191101 (2008). DOI 10.1103/PhysRevLett.100.191101

108. L. Baiotti, B. Giacomazzo, L. Rezzolla, Class. Quant. Grav. 26, 114005 (2009). DOI 10.1088/0264-9381/26/11/114005

109. J.G. Baker, J. Centrella, D.I. Choi, M. Koppitz, J. van Meter, Phys. Rev. D73, 104002 (2006). DOI 10.1103/PhysRevD.73.104002

110. A. Tchekhovskoy, J.C. McKinney, R. Narayan, Mon. Not. Roy. Astron. Soc. 379, 469 (2007). DOI 10.1111/j.1365-2966.2007.11876.x

111. C. Palenzuela, L. Lehner, O. Reula, L. Rezzolla, (2008). arXiv:0810.1838

112. M. Dumbser, O. Zanotti, (2009). arXiv:0903.4832

113. D. Neilsen, E.W. Hirschmann, R.S. Millward, Class. Quant. Grav. 23, S505 (2006). DOI 10.1088/0264-9381/23/16/S12

114. M. Anderson, E. Hirschmann, S.L. Liebling, D. Neilsen, Class. Quant. Grav. 23, 6503 (2006). DOI 10.1088/0264-9381/23/22/025

115. R. Oechslin, S. Rosswog, F. Thielemann, Phys.Rev. D 65, 103005 (2002)

116. D.J. Price, S. Rosswog, Science 312(5774), 719 (2006). DOI 10.1126/science.1125201

117. S. Rosswog, (2009). arXiv:0907.4890

118. S. Bonazzola, L. Villain, M. Bejger, Class. Quant. Grav. 24, S221 (2007). DOI 10.1088/0264-9381/24/12/S15

119. B. Costa, W.S. Don, Journal of Computational and Applied Mathematics 204(2), 209 (2007). DOI DOI: 10.1016/j.cam.2006.01.039

120. B. Costa, W.S. Don, Journal of Computational Physics 224(2), 970 (2007). DOI DOI: 10.1016/j.jcp.2006.11.002

121. W. Kastaun, Phys. Rev. D74, 124024 (2006). DOI 10.1103/PhysRevD.74.124024

122. M.D. Duez, P. Marronetti, S.L. Shapiro, T.W. Baumgarte, Phys. Rev. D67, 024004 (2003). DOI 10.1103/PhysRevD.67.024004

123. Y.T. Liu, S.L. Shapiro, Z.B. Etienne, K. Taniguchi, Phys. Rev. D78, 024012 (2008). DOI 10.1103/PhysRevD.78.024012

124. B. Giacomazzo, L. Rezzolla, L. Baiotti, (2009). arXiv:0901.2722

125. K. Kiuchi, M. Shibata, S. Yoshida, Phys. Rev. D78, 024029 (2008). DOI 10.1103/PhysRevD.78.024029

126. J. Braithwaite, A. Nordlund, A\&A 450(3), 1077 (2006). DOI 10.1051/00046361:20041980. URL http://dx.doi.org/10.1051/0004-6361:20041980

127. J. Braithwaite, MNRAS 386, 1947 (2008) 
128. J. Braithwaite, MNRAS 397, 763 (2009)

129. G. Toth, J. Comput. Phys. 161, 605 (2000)

130. E. Schnetter, C.D. Ott, G. Allen, P. Diener, T. Goodale, T. Radke, E. Seidel, J. Shalf, in Petascale Computing: Algorithms and Applications, ed. by D.A. Bader (Chapman \& Hall/CRC Computational Science Series, 2007), chap. 24

131. B. Zink, (2008). arXiv:0810.5349

132. B.D. Farris, T.K. Li, Y.T. Liu, S.L. Shapiro, Phys. Rev. D78, 024023 (2008). DOI 10.1103/PhysRevD.78.024023

133. M.D. Duez, Y.T. Liu, S.L. Shapiro, B.C. Stephens, Phys. Rev. D69, 104030 (2004). DOI 10.1103/PhysRevD.69.104030

134. N. Andersson, G.L. Comer, Living Rev. Rel. 10, 1 (2005)

135. Numerial INJection Analysis project. URL http://www.ninja-project.org

136. B. Aylott, et al., Class. Quant. Grav. 26, 165008 (2009). DOI 10.1088/02649381/26/16/165008

137. URL http://www.ninja-project.org/

138. K. Danzmann, A. Rüdiger, Classical Quantum Gravity 20, S1 (2003). URL stacks.iop.org/CQG/20/S2 